\title{
Interaction between stem cell factor and endothelin-1: effects on melanogenesis in human skin xenografts
}

\author{
Penkanok Sriwiriyanont ${ }^{1, *}$, Atsushi Ohuchi ${ }^{2, *}$, Akira Hachiya ${ }^{2,3}$, Marty O Visscher ${ }^{1}$ \\ and Raymond E Boissy ${ }^{1,4}$ \\ ${ }^{1}$ Skin Sciences Institute, Cincinnati Children's Hospital Medical Center, Cincinnati, OH, USA; ${ }^{2}$ Kao \\ Biological Science Laboratories, Haga, Tochigi, Japan; ${ }^{3}$ Department of Dermatology, Tokyo Medical \\ University, Tokyo, Japan and ${ }^{4}$ Department of Dermatology, University of Cincinnati College of Medicine, \\ Cincinnati, OH, USA
}

\begin{abstract}
The two paracrine melanogenic cytokines, stem cell factor (SCF) and endothelin-1 (ET-1), have been demonstrated to play pivotal roles in skin pigmentation including UVB-induced pigmentation and senile lentigo. However, little is known regarding their interactive effect on skin pigmentation. In order to investigate their roles in vivo, facultative pigmentation of human skin xenografts on severe combined immunodeficient (SCID) mice was assessed. After 1 week of acclimation in a pathogen-free barrier, dermatomed fresh cadaveric skin was surgically grafted onto the back of the mice and allowed to heal for 5-6 weeks prior to cytokine administration. Intradermal injections of SCF at 0.7 or $2.0 \mu \mathrm{g}$ significantly increased skin pigmentation when compared to vehicle control. Despite the lack of a dose-dependent pigmentation response following ET-1 administration, the combination of $0.2 \mu \mathrm{g} \mathrm{SCF}$ and $0.1 \mu \mathrm{g} \mathrm{ET}-1$ demonstrated a statistically significant increase in tyrosinase gene expression substantiated by the enhancement of melanin content and skin pigmentation compared to treatment with SCF alone or ET-1 alone. These findings establish an in vivo interaction between SCF and ET-1 with regard to their capacity to effect an increase in skin pigmentation.
\end{abstract}

Laboratory Investigation (2006) 86, 1115-1125. doi:10.1038/labinvest.3700469; published online 28 August 2006

Keywords: c-kit; endothelin-1; melanin; skin; stem cell factor; xenograft

Pigmentary disorders can arise through either hypoor hyper-proliferation of melanocytes secondary to genetic anomalies, postinflammatory reactions following skin wounding, exposure to UV radiation, or administration of various drugs. Many studies have demonstrated that melanocyte growth and survival are regulated by keratinocyte-derived factors, such as basic fibroblast growth factor (bFGF), stem cell factor (SCF) and endothelins (ET). Stem cell factor is a peptide growth factor that is related to haematopoiesis, melanogenesis and gametogenesis. ${ }^{1-3}$ As SCF is secreted from several cell types (eg keratinocytes, fibroblasts and endothelial cells), it has been hypothesized to play a critical role in cutaneous

Correspondence: Dr RE Boissy, PhD, Department of Dermatology, University of Cincinnati College of Medicine, 231 Albert Sabin Way ML-0592, Cincinnati, OH 45267-0592, USA.

E-mail: boissyre@ucmail.uc.edu

* These authors contributed equally to this work.

Received 17 March 2006; revised and accepted 11 July 2006; published online 28 August 2006 physiology and pathology. ${ }^{4}$ Activation of its transmembrane tyrosine kinase receptor, c-kit, promotes dimerization, autophosphorylation and transphosphorylation of several substrates at specific tyrosine residues, which serve as docking sites for src-homology-2 (SH2) domain-containing signal molecules. ${ }^{5}$ After associating with the adaptor protein, Grb2, an activated c-kit induces tyrosinase phosphorylation of phospholipase C- $\gamma$ (PLC- $\gamma$ ), which mediates the production of diacylglycerol and activates the protein kinase C (PKC) pathway. ${ }^{5,6}$ In addition, the binding of c-kit and p85 $\alpha$ induces tyrosine phosphorylation of inositide-3'-kinase (PI-3'-kinase), which may involve a negative feed back loop via serine phosphorylation in the PKC pathway. ${ }^{5}$ These multiple intracellular signaling molecules orchestrate the modulation of melanin biosynthesis and melanocyte proliferation.

The multiple functions of SCF/c-kit in mediating of epidermal cell adhesion, migration and proliferation support a role for these cytokines in melanoma development. Recent studies demonstrate that c-kit 
is downregulated during melanoma transformation and that the inhibition of c-kit hinders communication between keratinocytes and other cell types, causing inappropriate tissue localization and disruption of integrin-mediated survival and proliferative signals. ${ }^{7,8}$ Concomitantly, the restoration of a c-kit promoter, with AP-2-binding sites, in human melanoma cells was shown to effectively suppress tumorigenicity. ${ }^{9}$ Moreover, inactivating mutation of c-kit occurs in piebald patients who suffer from depigmentation of multiple body sites. ${ }^{10,11}$

Chronic UV exposure is linked to numerous skin pathologies (eg skin cancer, photoaging and immune damage). Short-term exposure to UV radiation stimulates epidermal cell proliferation and induces the secretion of several cytokines, including bFGF, $\alpha$-melanocyte stimulating hormone, nitric oxide, SCF and endothelin-1 (ET-1). Similar to SCF, ET-1 together with its receptor $\left(\mathrm{ET}_{\mathrm{B}} \mathrm{R}\right)$, utilize a common signaling pathway to regulate melanin synthesis and proliferation. ET influence several melanocyte functions including proliferation, dendrite formation and melanin synthesis. ${ }^{12}$ Both the SCF/c-kit and the ET-1/ET ${ }_{\mathrm{B}} \mathrm{R}$ pathways are crucial not only during neural crest formation and early melanocyte development, but also for melanocyte function postnatally. ${ }^{13-16}$ The migration, proliferation and differentiation of melanocytes in human hair follicles resemble the activities of melanoblasts during embryogenesis and suggest a critical role of SCF/ c-kit in the pigmentary unit during hair follicle regeneration. ${ }^{17}$ The functions of SCF/c-kit and ET-1/ $\mathrm{ET}_{\mathrm{B}} \mathrm{R}$ in skin pigmentary system have also been widely studied. Intradermal injection of SCF or c-kit neutralizing antibody into human xenografts was shown to regulate the number of Ki67-postive melanocytes. ${ }^{14}$ In addition, mutations of the ET-1 receptor result in cutaneous and ocular hypopigmentation (ie, Waardenburg Syndrome Type IV). ${ }^{13}$

The synergistic effect of these two paracrine melanogenic cytokines in enhancing the level of DNA synthesis and activating MAPK pathway was previously evaluated using an in vitro system, in which the combination of these two cytokines was added to human melanocyte cultures. ${ }^{18,19}$ Furthermore, both SCF/c-kit and ET-1/ET ${ }_{\mathrm{B}} \mathrm{R}$ pathways have been suggested to play roles in senile lentigo, the benign hyperpigmented macules that are commonly found in photoaged skin..$^{20,21}$ Quantitative reverse transcription-polymerase chain reaction (RT-PCR) and immunohistological analyses indicate that these pathways are stimulated in senile lentigo compared to noninvolved regions. These findings support the hypothesis that SCF and ET-1 synergistically stimulate melanogenesis. In order to evaluate the effects of these cytokines in living intact human skin, we established a xenograft model using fresh Caucasian cadaveric skin on ICR-SCID mice. Intradermal injections of the combination of SCF and ET-1 were evaluated for their effects on melanocyte size and numbers and their ability to upregulate tyrosinase activity and melanin synthesis, resulting in the facultative induction of skin pigmentation, in vivo.

\section{Materials and methods}

\section{Xenografting}

One week after acclimation in a pathogen-free barrier, 4- to 5-week-old female albino ICR-SCID mice (Taconic, Hudson, NY, USA) were surgically grafted with fresh split-thickness Caucasian type III skin, which was obtained 1-3 days after death (US Tissue and Cells, Cincinnati, OH, USA). After the mice were anesthetized with a mixture of isofluorane and oxygen, the endogenous dorsal skin was excised producing a wound bed of approximately $2-3 \mathrm{~cm}$ in diameter. A xenograft was then sutured in place with 6-0 nylon monofilament, (Ethicon Endo-Surgery, Cincinnati, OH, USA). The edges of the graft bed were treated with $0.5 \%$ Sensorcaine (Astrazeneca Pharmaceutical LP, Wilmington, DE, USA) to provide local analgesia. Following the surgical procedure, the mice were maintained in an incubator at $37^{\circ} \mathrm{C}$ until they fully recovered from the anesthesia.

\section{Cytokine Injections}

Human recombinant SCF (Peprotech, Rocky Hill, NJ, USA) and human recombinant ET-1 (Sigma Chemical Co., St Louis, MO, USA) were diluted either individually or in combination in PBS with $0.1 \%$ bovine serum albumin (BSA). Upon confirmation of complete healing, either SCF $(0.2,0.7$ or $2.0 \mu \mathrm{g})$ or ET-1 (0.1, 0.3 or $1.0 \mu \mathrm{g})$ was intradermally injected into the xenograft on three consecutive days using a 30 gauge-insulin syringe. Animals were killed 4 days postinjection, and skin biopsies were collected for quantitative analyses (Figure 1). To determine if the two cytokines interact synergistically to affect cutaneous pigmentation, SCF was injected alone at $0.2 \mu \mathrm{g}$ or in combination with $0.1 \mu \mathrm{g}$ ET-1 following the same injection protocol as previously described.

\section{Skin Surface Imaging Analysis}

In situ digital magnified (10X) images of grafted skin were obtained using a Charm View ${ }^{\mathrm{TM}}$ digital image capture device (Moritex, Tokyo, Japan). This device was used in the back-scattering illumination mode to eliminate interfering specular interference from the skin surface. Images were obtained before the first cytokine injection and immediately prior to killing. Pigmentation levels were determined from the images using the L-a-b color measurement scale in Adobe Photoshop ${ }^{\circledR}$ to obtain L-scale values. The L-scale is a measure of image lightness with values ranging from 0 (black) to 100 (white). 


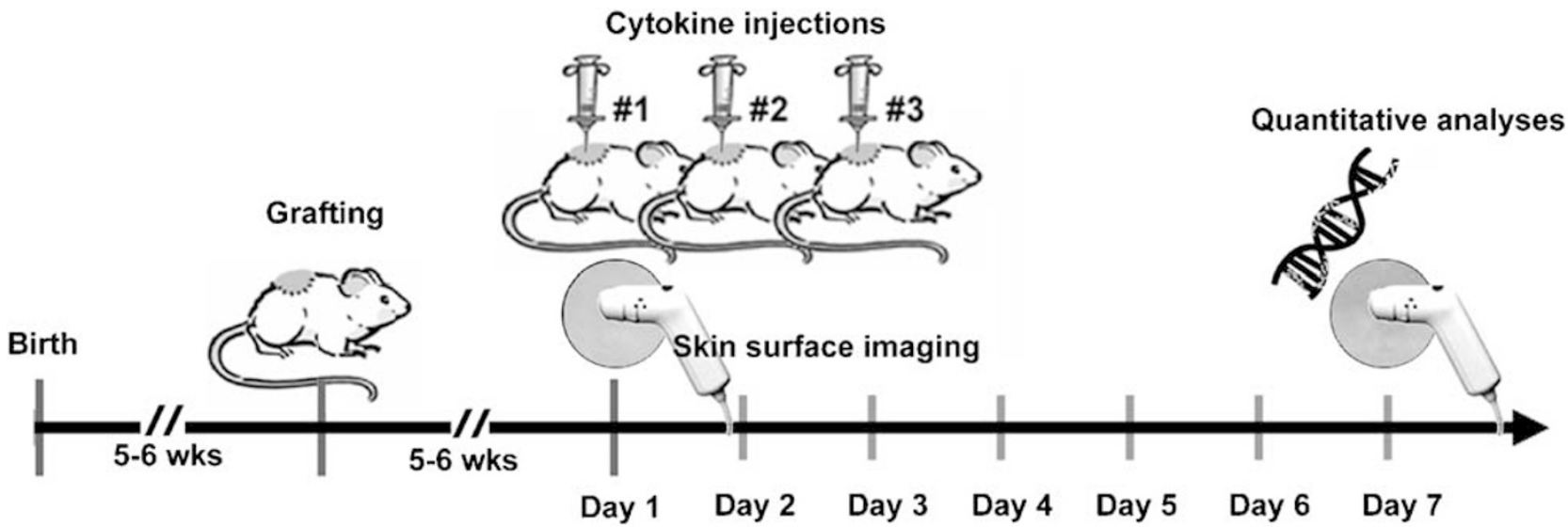

Figure 1 Experimental timeline. Five to six week old ICR-SCID mice were grafted with cadaveric skin as described in 'Materials and methods'. Grafts were permitted to heal and stabilize over the following 5-6 weeks. The skin surface was imaged periodically during healing, immediately prior to the first cytokine injection and again on the day of sacrifice. Cytokine were administered for 3 consecutive days. The mice were sacrificed 4 days after the final injection and biopsies were collected for L-DOPA staining, immunohistochemistry and real time qRT-PCR.

\section{L-DOPA Staining}

Epidermal sheets were separated from dermis after incubating whole skin specimens with $2 \mathrm{M}$ sodium bromide in PBS for $0.5-1.0 \mathrm{~h}$ at $37^{\circ} \mathrm{C}$. Activated melanocytes were detected by incubating the epidermal sheets in PBS containing $1.0 \mathrm{mg} / \mathrm{mL}$ of L-DOPA substrate (Sigma Chemical Co., St Louis, MO, USA) for $8 \mathrm{~h}$ or overnight. The epidermal sheets were then mounted on to glass microscope slides for subsequent analysis by light microscopy.

\section{Immunohistochemistry}

Rabbit antisera against human c-kit receptor (IBL, Japan), mouse monoclonal antibody against human fibroblasts-CD90 and Thy-1 (EMD Biosciences, San Diego, CA, USA) and mouse monoclonal antibodies against human gp100/pmel17 (HMB45; Dako, Carpinteria, CA, USA) were used as primary antibodies. Immunoreactivity in paraffin sections was detected using the avidin-biotin peroxidase complex method (Vector Laboratories, Burlingame, CA, USA) and AEC substrate kit (Vector Laboratories) after heat pretreatment in $10 \mathrm{mM}$ citrate buffer. Negative controls were performed using either normal rabbit IgG or normal mouse IgG. All sections were counterstained with hematoxylin (Zymed, South San Francisco, CA, USA).

\section{Real-Time Quantitative Reverse Transcription Polymerase Chain Reaction}

Total RNA was extracted from paraffin sections using the Absolutely RNA FFPE kit (Strategene, La Jolla, CA, USA) according to the manufacturer's instructions. In brief, two of $5 \mu \mathrm{m}$ paraffin sections were deparaffinized in L-lemonene, and rehydrated by serial dilution of ethanol in DNase-RNase-free water. Total RNA was extracted using a spin column, and the yield of total RNA from each sample was determined photospectrometrically at $260 \mathrm{~nm}$. RNA quality, determined by measurement of the 260:280 absorbance ratio, ranged between 1.6 and 2.0 . Approximately $100 \mathrm{ng}$ total RNA was converted to cDNA using the cDNA Achieve kit (Applied Biosystems, Foster City, CA, USA). Tyrosinase (TYR) transcript expression was normalized against glyceraldehyde-3-phosphate dehydrogenase (GAPDH) using Taqman $^{\mathrm{TM}}$ Gene Expression assays, which have primers lengths $<100 \mathrm{bps}$.

\section{Statistical Analysis}

Statistically significant differences between treatment and control groups were analyzed using nonparametric one-way analysis of variance (ANOVA). Whenever appropriate, the multiple pairwise test, Student-Newman-Keuls (SNK) was used to evaluate difference between groups. Differences were considered statistically significant if $P<0.05$.

\section{Results}

\section{Wound Healing after Xenografting}

At 1 week postsurgery, a weak localized inflammatory response was noted in the xenografts as indicated by the presence of mild erythema and slight swelling (Figure 2a). Also at 1 week, neovascularization was detected by the presence of small blood vessels in the papillary dermis (data not shown). Typically, by 2 weeks, a slight shrinkage of the xenograft was observed (Figure 2b). Wound healing was judged to be completed by $5-6$ weeks and after this period of recovery, graft color and graft 

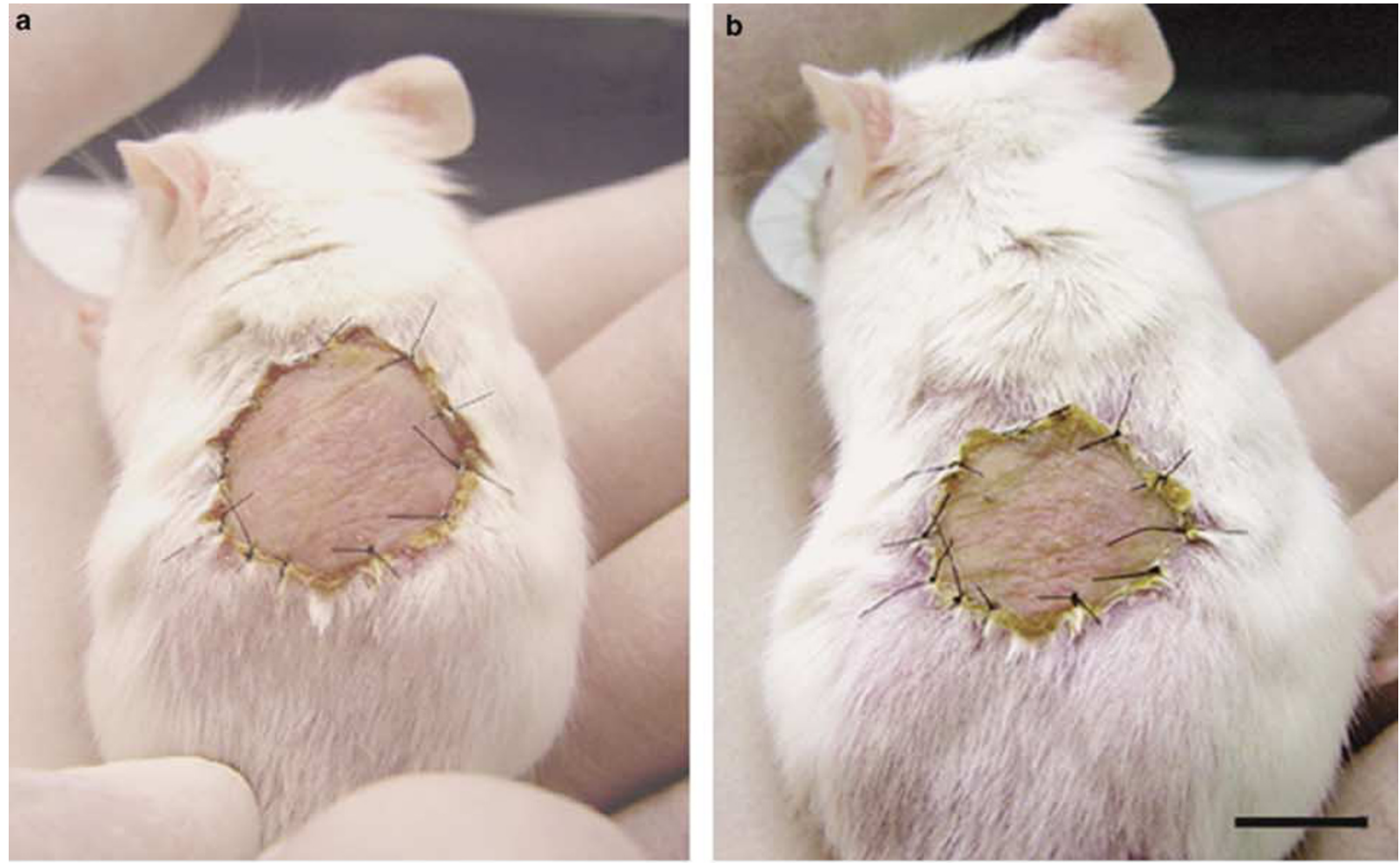

C

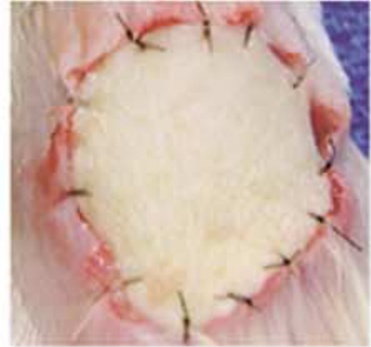

Day 1

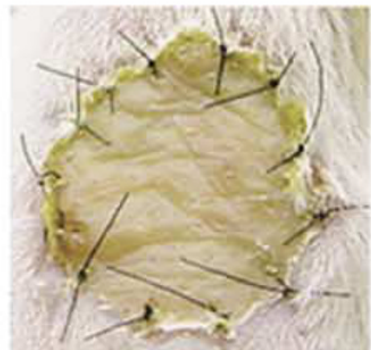

Day 15

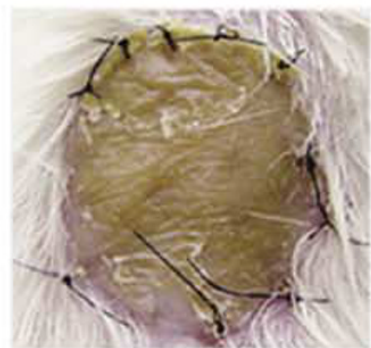

Day 27

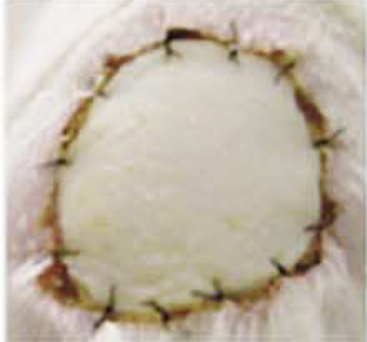

Day 3

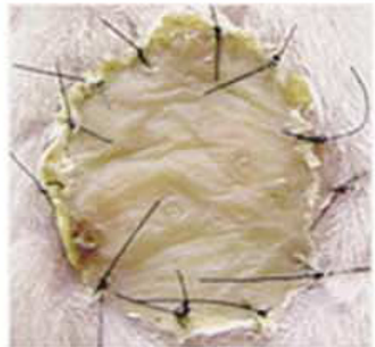

Day 17

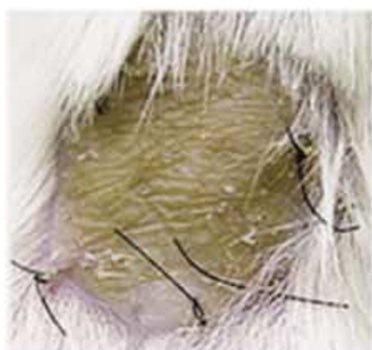

Day 31

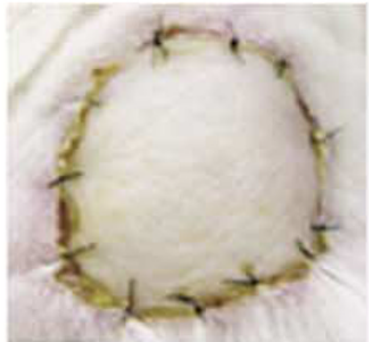

Day 6

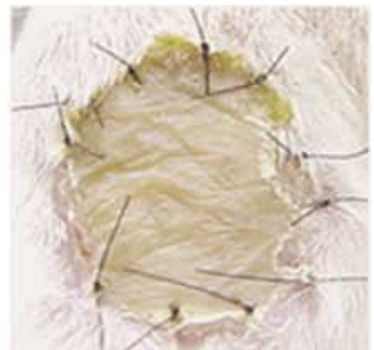

Day 20

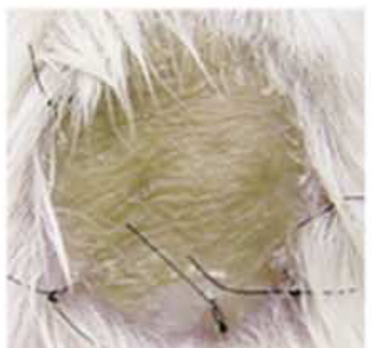

Day 34

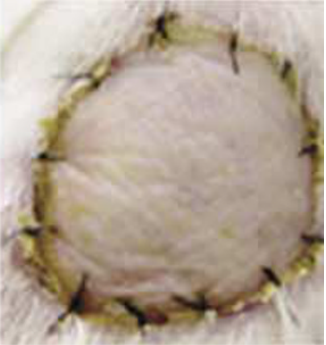

Day 8

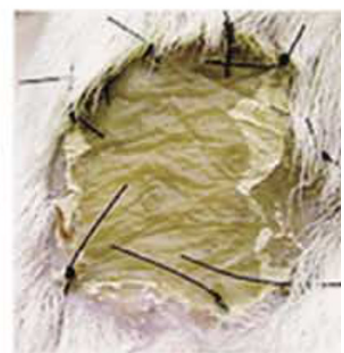

Day 22

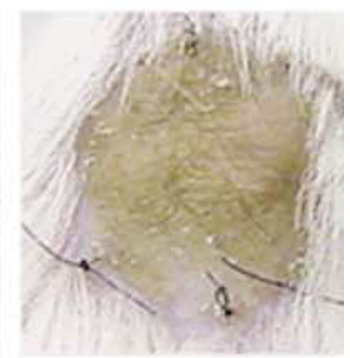

Day 41 
size remained stable (Figure 2c). Immunohistochemical analysis of a representative xenograft using mouse monoclonal antibodies against human fibroblasts demonstrated a clear and distinct junction between the grafted human skin and the skin of the murine host (Figure 3a). In addition, skin sections stained using the Fontana-Masson technique demonstrated the presence of melanin in the overlying human epidermis but not in the surrounding albino murine skin (Figure 3b).

\section{Effect of SCF and/or ET-1 on Skin Coloration}

Either SCF or ET-1 was injected intradermally into the human skin xenograft according to the dose and schedule described in 'Materials and methods'. Four days after the last injection, L-scale measurement of digital images of the grafted skin revealed a dosedependent hyperpigmentation localized to the sites of SCF injection (Figure 4; left panel). In contrast, administration of ET-1 resulted in a moderate, but statistically insignificant increase in overall skin pigmentation (Figure 4; right panel). In order to determine whether or not the combination of SCF and ET-1 would interact to produce a further increase in pigmentation, another set of mice were injected intradermally with SCF alone $(0.2 \mu \mathrm{g})$, ET-1 alone $(0.1 \mu \mathrm{g})$, or SCF and ET-1 in combination $(0.2 \mu \mathrm{g}$ SCF $+0.1 \mu \mathrm{g}$ ET-1) for three consecutive days.
As observed previously, the administration of SCF alone at $0.2 \mu \mathrm{g}$ or ET- 1 alone at $0.1 \mu \mathrm{g}$ resulted in only a slight increase in the pigmentation. However,
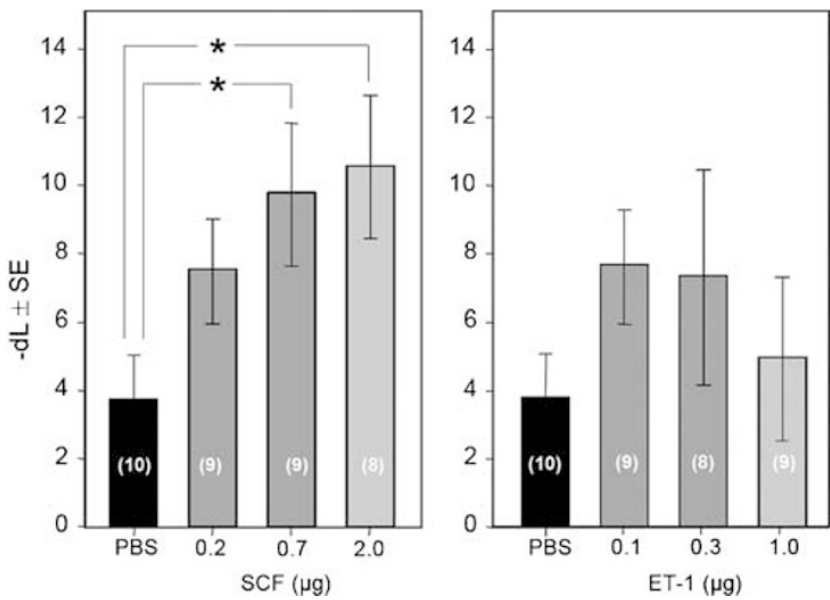

Figure 4 Effect of SCF or ET-1 treatment on skin pigmentation The effect of either SCF injection or ET-1 injection on skin pigmentation was monitored by L-scale measurement before the initial cytokine injection and 4 days after the final injection. Data are depicted as the difference in the L-score between the first and last measurements. Treatment with SCF resulted in a dosedependent, statistically significant increase in skin pigmentation over the measurement interval $(P<0.05)$. Pigmentation in the ET1-treated grafts increased slightly over this time period. However, the observed change was not statistically significant. Data are reported as mean \pm s.e., $n=8-10$ as indicated in parentheses. $*=P<0.05$.
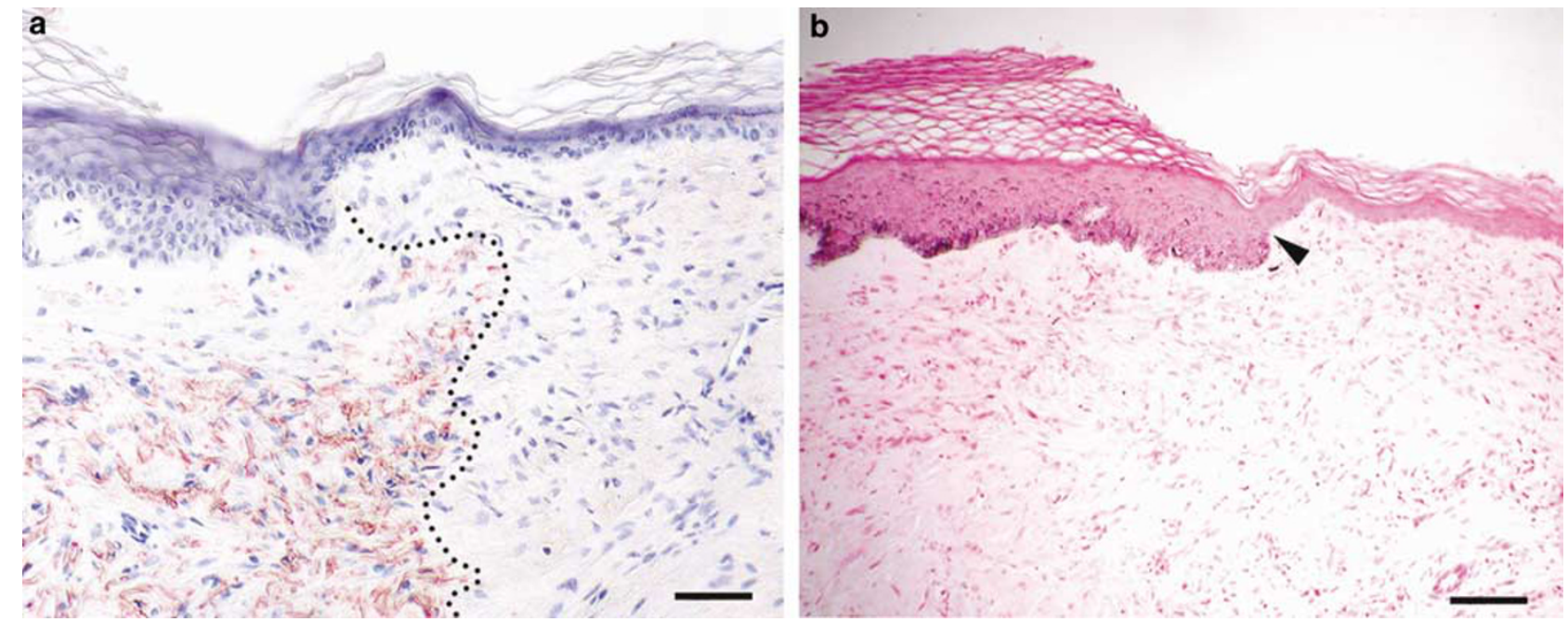

Figure 3 Human-murine junction in the xenograft model. A representative xenograft is depicted at 10 weeks post surgery. Immunohistochemical staining using mouse antisera against human fibroblasts (CD90 and Thy-1) indicates a distinct dermal junction with a corresponding change in epidermal thickness (a). Concomitantly, melanin is present within the epidermis in the grafted skin but not in the epidermis of the albino murine host as demonstrated by Fontana Masson staining of the same histological specimen (b). The scale bar denotes $100 \mu \mathrm{m}$ in both panels.

Figure 2 Surface appearance of the xenografts. At 1 week after the grafting procedure, a weak transient inflammatory reaction was typically observed in the xenografted skin (a). By 2 weeks postsurgery, the cutaneous inflammation had subsided and the size of the graft was moderately reduced; scale bar $=10 \mathrm{~mm}(\mathbf{b})$. Graft size continued to decrease slightly over the next few weeks but remained stable after 5-6 weeks. As demonstrated with another xenograft, the bottom portion of this figure illustrates the progressive hyperpigmentation that was characteristic during xenograft healing and adaptation (c). After 1 month, this hyperpigmentation ceased and skin color remained stable. 


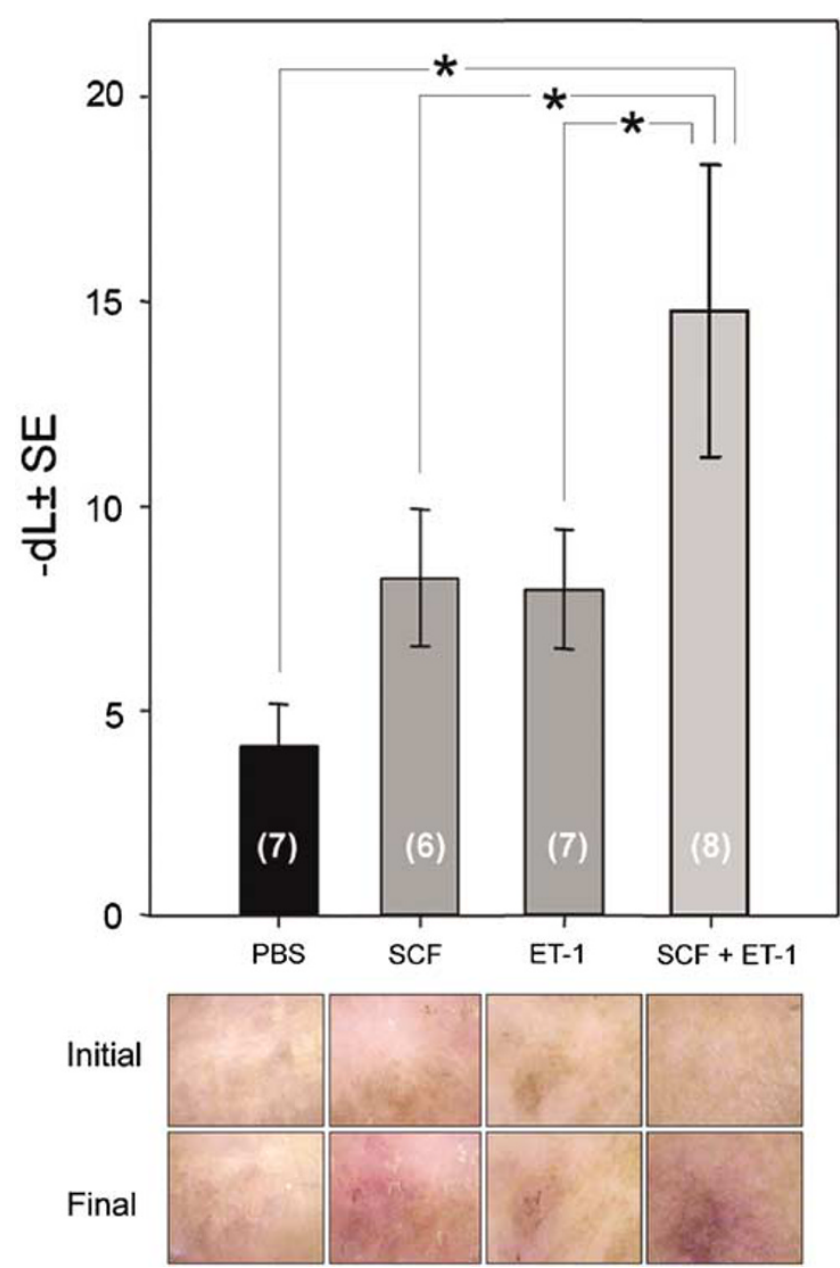

Figure 5 Effect of combined SCF and ET-1 treatment on skin pigmentation. In this set of experiments, the combination of SCF $(0.2 \mu \mathrm{g})$ and ET-1 $(0.1 \mu \mathrm{g})$ was compared to the same dose of these cytokines injected individually. As described in the previous figure, L-scale measurements were used to monitor skin pigmentation. The data reflect the difference between the initial and final L-score measurements, and are reported as mean \pm s.e., $n=6-8$ as indicated in parentheses. The upper portion of this figure shows that only the combined cytokine treatment resulted in a statistically significant increase in skin pigmentation $(P<0.05)$. The lower portion of the figure shows the images from which the L-scale data were derived. While there is a slight erythema following repeated injection of SCF, only the combined cytokine treatment resulted in significant hyperpigmentation. ${ }^{*}=P<0.05$.

the combination of ET-1 and SCF at these same levels induced a statistically significant increase in pigmentation relative to vehicle controls and to either cytokine administered alone (Figure 5).

\section{Immunoreactivity of Antibodies against c-kit Receptor and Human Melanosomes}

The effect of SCF and ET-1 on cutaneous pigmentation was also illustrated using paraffin sections obtained 4 days following the last cytokine injection. Cells staining positive for c-kit were observed in the xenografts along the basal epidermal layer and within the superficial dermis (Figure 6). Regardless of treatment regimen, the amount of c-kit expression in the epidermis was relatively equivalent. Conversely, after injection of either SCF alone or SCF with ET-1, the number of c-kit positive cells in the dermis was more pronounced.

Immunostaining of the xenografts with the antibody HMB-45 that binds to the human melanosomal matrix protein (gp100/pmel17) showed an increase in the number of basal melanocytes following all of the cytokine treatment regimens compared to vehicle-treated controls (Figure 7). Notably, the melanocyte populations in the xenografts treated with either SCF or SCF in combination with ET-1 demonstrated a slight hypertrophy, which was associated with an accompanying increase in dendricity.

\section{Melanocyte Assessment}

To further investigate the observed cutaneous hyperpigmentation, whole intact epidermal sheets were isolated from the xenografts 4 days after the final cytokine injection and subsequently stained with melanocyte-specific DOPA stain. These specimens were used to assess differences in the shape and number of DOPA-positive melanocytes in the xenografts. SCF given alone or in combination with ET-1 induced melanocyte hypertrophy (Figure 8; top panel). Additionally, when compared to vehicle controls, a statistically significant increase in the number of DOPA-positive cells was found in xenografts treated with SCF in combination with ET-1 (Figure 8; bottom panel).

\section{Tyrosinase Gene Expression}

Real-time quantitative RT-PCR analysis of human skin grafts was performed to determine comparative levels of TYR mRNA transcripts. There was no significant difference in the levels of transcript expression between vehicle-treated control grafts and grafts that were injected with either $0.2 \mu \mathrm{g}$ SCF or $0.1 \mu \mathrm{g}$ ET-1 (Figure 9). Interestingly, the combined injection of $0.2 \mu \mathrm{g}$ SCF and $0.1 \mu \mathrm{g}$ ET resulted in a synergistic and statistically significant enhancement in the expression of this transcript (Figure 9).

\section{Discussion}

Many studies have demonstrated the benefit of using xenografted SCID mice as a chimeric model in studying human skin diseases. ${ }^{22-24}$ Similar gene expression patterns in xenografts, pre- and postgrafting onto SCID mice, indicate that this model maintains much of its original tissue function and that the host mouse lacks an immune response that could influence cytokine- or chemokine-based immune therapies. ${ }^{24}$ In our preliminary grafting 

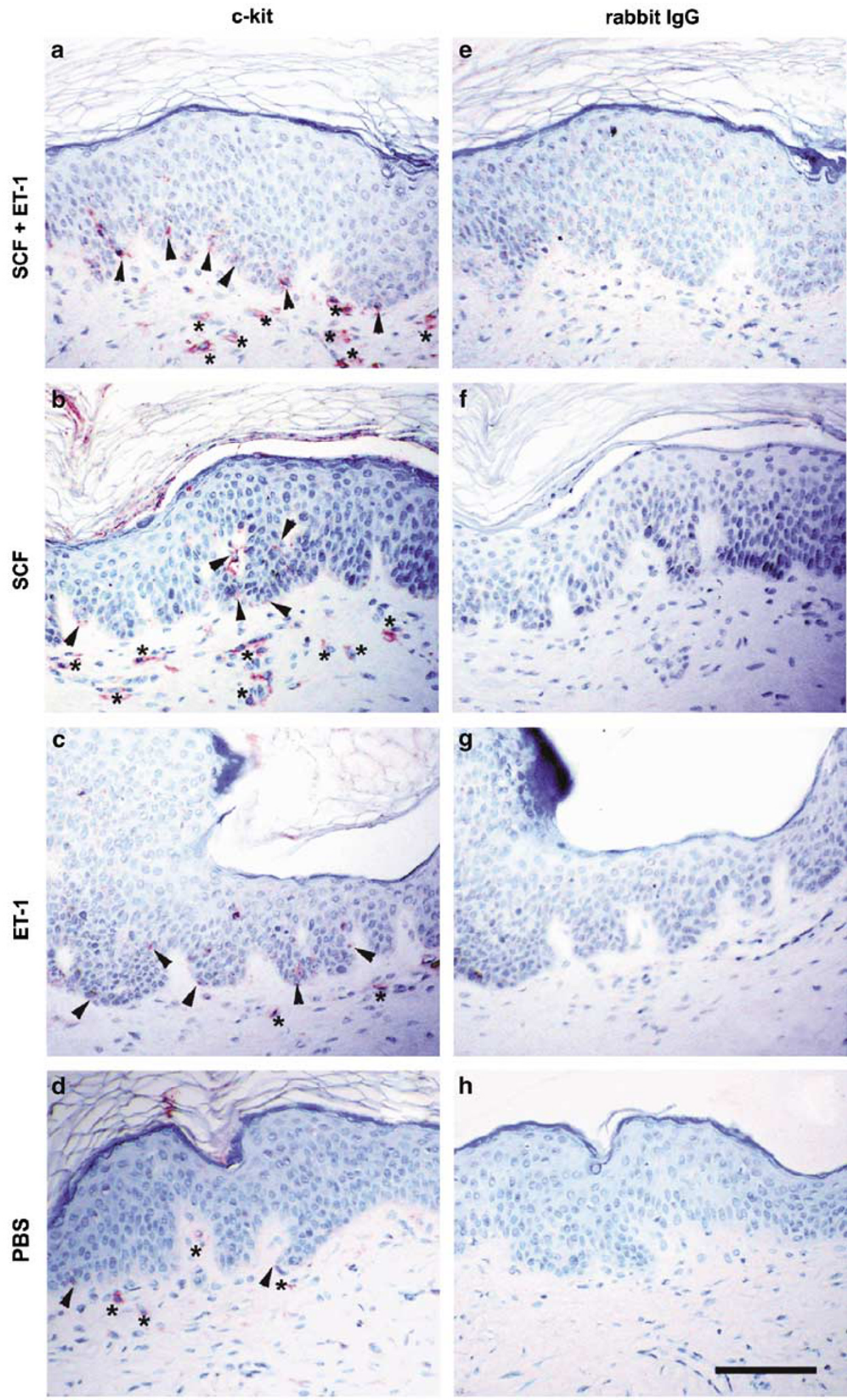

Figure 6 C-kit localization in epidermis and dermis following cytokine treatment. Xenografts were treated with SCF and ET-1 (a, e), SCF $(\mathbf{b}, \mathbf{f})$, ET-1 (c, g) or PBS (d, h) as described in Materials and methods. Cytokine injected xenografts were biopsied and immunohistological analysis was performed using c-kit antibody (a-d) or non-specific rabbit IgG $(\mathbf{e}-\mathbf{h})$. Cytokine-treated xenografts demonstrated slightly increased basal epidermal expression of c-kit relative to the vehicle-injected controls (arrows). While there was no observable difference in c-kit expression between any of the cytokine treated groups in the epidermis, there was an increase in the number of dermal c-kit positive cells after treatment with either SCF alone or SCF combined with ET-1 (asterisks) (scale bar $=100 \mu \mathrm{m}$ ). 

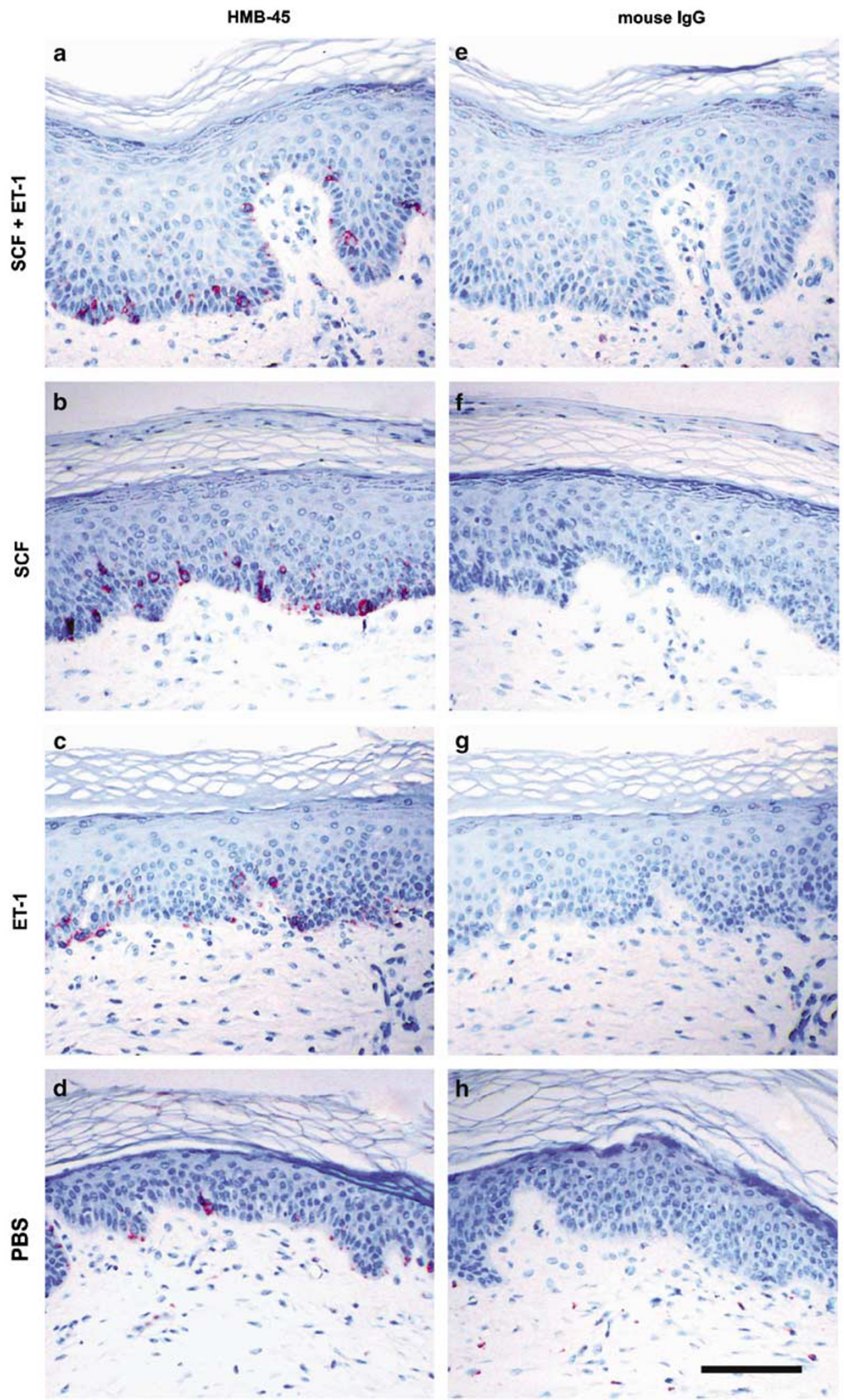

Figure 7 Melanocyte localization following cytokine treatment. Xenografts were treated with SCF and ET-1 (a, e), SCF (b, f), ET-1 $(\mathbf{c}, \mathbf{g})$ or PBS $(\mathbf{d}, \mathbf{h})$ as described in Materials and methods. These photomicrographs show the localization of melanocytes by immunohistochemical staining with HMB-45 antibody (a-d) or non-specific mouse IgG (e-h). All of the cytokine treated xenografts demonstrated an increase in the number of melanocytes over the vehicle-treated control. While it is difficult to discern if there is a definitive difference in the number of melanocytes between the cytokine treated groups, the dendricity of the melanocytes appears to be greater in both SCF treated and SCF:ET-1-treated grafts (scale bar $=100 \mu \mathrm{m}$ ). 

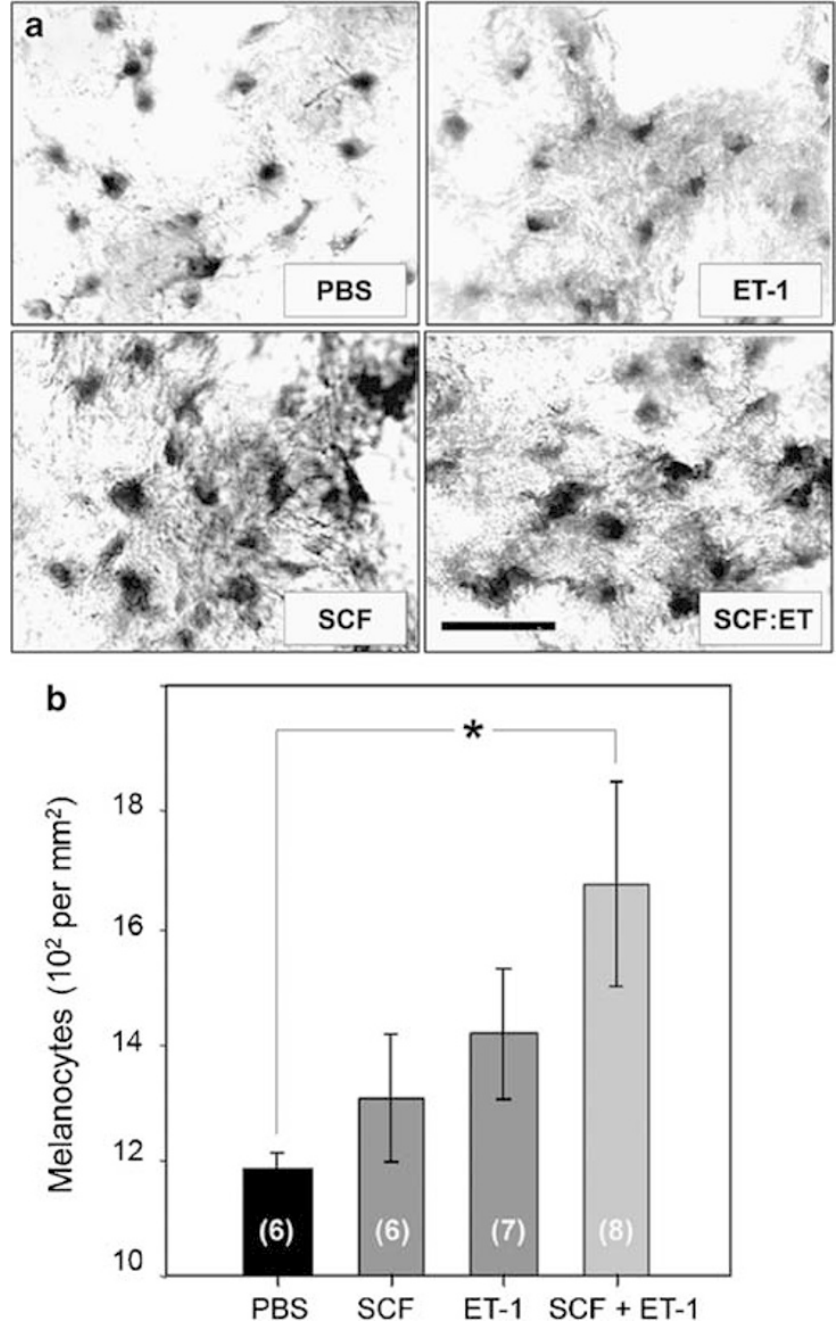

Figure 8 Effect of SCF and/or ET-1 treatment on melanocyte size, shape and number. The upper portion of this figure shows representative epidermal sheets from each experimental group of grafted human skin following treatment with PBS (vehicle), SCF $(0.2 \mu \mathrm{g})$, ET-1 $(0.1 \mu \mathrm{g})$, or the combination of both cytokines (scale bar $=100 \mu \mathrm{m}$ ). When SCF was injected alone or in combination with ET-1, xenograft melanocytes demonstrated hypertrophy and increased dendriticity (a). The DOPA-positive melanocytes were quantified and normalized to surface area. These data are displayed graphically in the lower portion of the figure as mean \pm s.e., $n=5-7$, as indicated in parentheses. Neither SCF nor ET-1 alone significantly increased the number of melanocytes; however, the combination of both cytokines did result in a statistically significant increase in melanocyte number (b). ${ }^{*}=P<0.05$

studies, we evaluated the advantages and disadvantages of using either full-thickness skin collected from breast reduction surgery or fresh split-thickness cadaveric skin. Our results indicated no discernable difference in either wound healing or in rejection rate of the xenografts (data not shown). Skin from either source initiated tissue vascularization within the first week after grafting and, in most instances; wounds were judged to be completely healed within 5-6 weeks. However, dermatomed cadaveric skin thickness is more uniform rendering

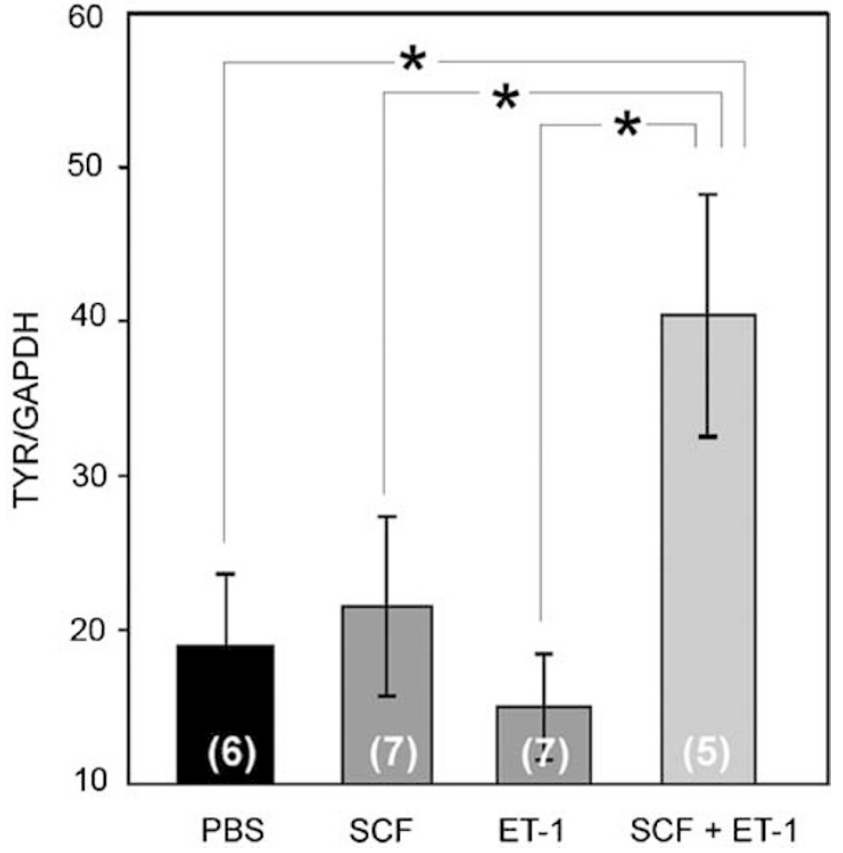

Figure 9 TYR gene expression following cytokine treatment. The expression of TYR transcript in the xenografts that were injected with PBS, SCF $(0.2 \mu \mathrm{g})$, ET-1 $(0.1 \mu \mathrm{g})$ or the combination of both cytokines was measured using real-time qRT-PCR normalized against GAPDH. Data are reported as mean \pm s.e., $n=5-7$, as indicated in parentheses. TYR expression was significantly upregulated in the combined cytokine treated grafts only. $*=P<0.05$

this split-thickness skin more practical for the grafting procedure. Furthermore, immunohistochemical staining of xenografts and adjacent tissue with mouse anti-human CD90 and Thy-1 antibodies indicated a distinct and stable boundary between graft and host. This result was also confirmed by localization of melanin within the graft, but not in the albino host tissue. The establishment of this stable and clearly observable delineation between human and murine skin strongly suggests that this SCID xenograft model could be a useful alternative to clinical studies performed on human skin.

After healing, either SCF or ET-1 was injected intradermally into the xenografts over 3 consecutive days. Digital photographs of the xenografts were obtained and an empirical color space analysis of the images was performed. L-scale analysis of these images showed a dose-dependent, statistically significant increase in pigmentation after SCF administration. In contrast, while ET-1 treatment elicited a slight hyperpigmentation, skin coloration was not significantly different than the vehicle-treated controls. The partial increase in pigmentation following ET-1 injection may be attributed to insufficient dosage or to insufficient duration of treatment. The concentrations of ET-1 that were evaluated in this study were based on the levels that were previously shown to be effective in increasing melanogenesis in culture. ${ }^{25}$ However, an effective pigment-inducing 
dose of ET-1 for intact in vivo skin has yet to be determined.

It is also possible that the partial hyperpigmentation observed after ET-1 treatment is due to the functional relationship between SCF and ET-1. It has been reported that after 2 minimal erythema doses of UVB, SCF mRNA transcripts, SCF protein expression and membrane bound-SCF localization were enhanced within 3 days, whereas levels of ET1 expression were not enhanced until 7 days post UVB exposure. ${ }^{15,26}$ The sequential expression of these two cytokines in human skin after exogenous stimuli suggests that the $E T-1 / \mathrm{ET}_{\mathrm{B}} \mathrm{R}$ pathway is downstream of the SCF/c-kit pathway, and that SCF has a higher hierarchy in UVB-induced pigmentation.

In the dosing regimen employed in this study, injection of either ET-1 $(0.1 \mu \mathrm{g})$ or injection of the lowest SCF dose $(0.2 \mu \mathrm{g})$ yielded a slight increase in graft pigmentation albeit one not statistically different from the vehicle-treated controls. These levels of ET-1 and SCF were then combined to determine whether or not these two cytokines would augment each other to elicit an increase in pigmentation. Lscale data collected from these grafts indicate that the combined cytokines increased graft pigmentation approximately 3.5-fold over vehicle-treated controls, a change that was statistically significant.

Examination and quantification of L-DOPA stained cells in epidermal sheets isolated from treated xenografts substantiated these L-scale data showing that melanocyte proliferation followed a similar response pattern. In a related analysis, TYR mRNA transcript levels were measured by qRT-PCR and normalized to the housekeeping transcript, GAPDH. In grafts that were treated with SCF and ET-1 in combination, tyrosinase mRNA levels were significantly greater than the transcript levels observed in grafts that were treated with either cytokine alone. Interestingly, tyrosinase mRNA transcript levels in grafts that were treated with both cytokines were also significantly greater than the additive transcript levels seen in grafts that were treated with each cytokine individually, suggesting that, with respect to TYR transcript levels, these two cytokines may act synergistically.

Recent studies have demonstrated that not only membrane bound-SCF, but also its soluble form can act as a stimulator of epidermal hyperpigmentation in mastocytosis. ${ }^{14,27,28}$ It has also been reported that repetitive subcutaneous injection of polyethylene glycol-modified SCF stimulated an increase in the tissue mast cell population, while cessation of SCF administration resulted in a reduction. ${ }^{29}$ Therefore, it was not surprising that, in our experiments, treatment with SCF resulted in an increase dermal c-kit-positive cells, presumably mast cells. While we did not attempt to quantify the increase in the putative mast cell population following cytokine treatment, immunohistochemical examination of the xenografts strongly suggested that treatment with SCF alone or in combination with ET-1 yielded an increase in the number of dermal c-kit positive cells. But unlike the interactive effect to increase melanocyte size and number that was observed in the epidermis, the inclusion of ET-1 with SCF resulted in no detectable change in the mast cell population.

It is noteworthy that whether pigmentation was determined by L-scale analysis, melanocyte number or tyrosinase expression, the increase observed following ET-1 treatment alone never reached the level of statistical significance while the increase observed following the combined treatment of SCF and ET-1 consistently achieved statistical significance. Taken together, these results strongly suggest that the interaction between SCF and ET-1 has a substantive effect on melanogenesis.

Previous studies have suggested that down-regulation of SCF in vitiliginous keratinocytes was primarily responsible for keratinocyte and melanocyte apoptosis. $^{30,31}$ The treatment with either SCF or ET-1 has been shown to influence cell adhesion and melanocyte cell migration in culture, implying the plausible role of these two cytokines in regulating melanocyte function in vitiligo. ${ }^{32,33}$ In addition, it has been postulated that these cytokines may influence the recruitment of melanocytes out of pigmented hair follicles in vitiligo lesions to induce repigmentation. ${ }^{33}$ Our findings demonstrate that SCF and ET-1 in combination induce proliferation, melanogenesis and dendritogenesis of melanocytes. These data suggest that SCF administered locally in combination with ET-1 may have a potential therapeutic effect for the treatment of melanocytopenic disorders such as vitiligo by inducing melanocyte migration, proliferation and restoration of function in the depigmented lesion.

\section{Acknowledgements}

We wish to acknowledge William Pickens and Steven Hoath for their review of the manuscript.

\section{References}

1 Besmer P, Manova K, Duttlinger R, et al. The kit-ligand and its receptor c-kit/w: pleiotropic roles in gametogenesis and melanogenesis. Dev Suppl 1993; 125-137.

2 Blume-Jensen P, Jiang G, Hyman R, et al. Kit/stem cell factor receptor-induced activation of phosphatidylinositol 3'-kinase is essential for male fertility. Nat Genet 2000;24:157-162.

3 Fujio K, Evarts RT, Hu Z, et al. Expression of stem cell factor and its receptor, c-kit during liver regeneration from putative stem cell in adult rat. Lab Invest 1994;70:511-516.

4 Grabbe J, Welker P, Dippel E, et al. Stem cell factor, a novel cutaneous growth factor for mast cells and melanocytes. Arch Dermatol Res 1994;287:78-84. 
5 Blume-Jensen P, Ronnstrand L, Gout I, et al. Modulation of Kit/stem cell factor receptor-induced signaling by protein kinase C. J Biol Chem 1994;269:2179321802.

6 Maddens S, Charruyer A, Plo I, et al. Kit signaling inhibits the sphingomyelin-ceramide pathway through PLC gamma 1: implication in stem cell factor radioprotective effect. Blood 2002;100:1294-1301.

7 Bendall LJ, Makrynikola V, Hutchinson A, et al. Stem cell factor enhances the adhesion of AML cells to fibronectin and augments fibronectin-mediated antiapoptotic and proliferative signals. Leukemia 1998;12: 1375-1382.

8 Lahav R. Endothelin receptor B is required for the expansion of melanocyte precursors and malignant melanoma. Int J Dev Biol 2005;49:173-180.

9 Huang S, Jean D, Luca M, et al. Loss of AP-2 results in downregulation of $C$-KIT and enhancement of melanoma tumorigenicity and metastasis. EMBO J 1998;17: 4358-4369.

10 Fleischman RA, Saltman DL, Stastny V, et al. Deletion of the $C$-kit protooncogene in the human developmental defect piebald trait. Proc Natl Acad Sci 1991; 88:10885-10889.

11 Spritz RA, Holmes SA, Ramesar R, et al. Mutations of the KIT (mast/stem cell growth factor receptor) protooncogene account on the continuous range of phenotypes in human piebaldism. Am J Hum Genet 1992;51: 1058-1065.

12 Demunter A, De Wolf-Peters C, Degreef $\mathrm{H}$, et al. Expression of the endothelin-B receptor in pigment cell lesions of the skin. Evidence for its role as tumor progression marker in malignant melanoma. Virchows Arch 2001;438:485-491.

13 Reid K, Turnley AM, Maxwell GD, et al. Multiple roles for endothelin in melanocyte development: regulation of progenitor number and stimulation of differentiation. Development 1996;122:3911-3919.

14 Grichnik JM, Burch JA, Burchette J, et al. The SCF/KIT pathway plays a critical role in the control of normal human melanocyte homeostasis. J Invest Dermatol 1998;111:233-238.

15 Hachiya A, Kobayashi A, Ohuchi A, et al. The paracrine role of stem cell factor/c-kit signaling in the activation of human melanocytes in ultraviolet-Binduced pigmentation. J Invest Dermatol 2001;116: 578-586.

16 Aoki H, Motohashi T, Yoshimura N, et al. Cooperative and indispensable roles of endothelin 3 and KIT signalings in melanocyte development. Dev Dyn 2005; 233:407-417.

17 Botchkareva NV, Khlgatian M, Longley BJ, et al. SCF/ c-kit signaling is required for cyclic regeneration of the hair pigmentation unit. FASEB J 2001;15:645-658.

18 Imokawa G, Yada Y, kimura M. Signalling mechanisms of endothelin-induced mitogenesis and melanogenesis in human melanocytes. Biochem J 1996;314:305-312.

19 Imokawa G, Kobayashi T, Miyagishi M. Intracellular signaling mechanisms leading to synergistic effects of endothelin-1 and stem cell factor on proliferation of cultured human melanocytes. J Biol Chem 2000; 275:33321-33328.

20 Kadono S, Manaka I, Kawashima M, et al. The role of the epidermal endothelin cascade in the hyperpigmentation mechanism of lentigo senilis. J Invest Dermatol 2001;116:571-577.

21 Hattori H, Kawashima M, Ichikawa $\mathrm{Y}$, et al. The epidermal stem cell factor is over-expressed in lentigo senilis: implication for the mechanism of hyperpigmentation. J Invest Dermatol 2004;122:1256-1265.

22 Takizawa Y, Saida T, Tokuda Y, et al. Engraftment of precursor lesions of human cutaneous neoplasms onto C.B-17 SCID mice: a useful in vivo experimental model of carcinogenesis in human skin. Arch Dermatol Res 1995;287:237-241.

23 Boehncke WH. The SCID-hu xenogeneic transplantation model: complex but telling. Arch Dermatol Res 1999;291:367-373.

24 Bankert RB, Egilmez NK, Hess SD. Human-SCID mouse chimeric models for the evaluation of anticancer therapies. Trends Immunol 2001;22:386-393.

25 Imokawa G, Miyagishi M, Yada Y. Endothelin-1 as a new melanogen: coordinated expression of its gene and the tyrosinase gene in UVB-exposed human epidermis. J Invest Dermatol 1995;105:32-37.

26 Hachiya A, Kobayashi A, Yoshida Y, et al. Biphasic expression of two paracrine melanogenic cytokines, stem cell factor and endothelin-1, in ultraviolet Binduced human melanogenesis. Am J Pathol 2004;165: 2099-2109.

27 Kunisada T, Lu SZ, Yoshida H, et al. Murine cutaneous mastocytosis induced by keratinocyte expression of transgenic stem cell factor. J Exp Med 1998;187: 1565-1573.

28 Longley Jr BJ, Morganroth GS, Tyrrell L, et al. Altered metabolism of mast-cell growth factor ( $c$-kit ligand) in cutaneous mastocytosis. N Engl J Med 1993;328: 1302-1307.

29 Maurer M, Galli SJ. Lack of significant skin inflammation during elimination by apoptosis of large numbers of mouse cutaneous mast cells after cessation of treatment with stem cell factor. Lab Invest 2004;84: 1593-1602.

30 Lee AY, Kim NH, Choi WI, et al. Less keratinocytederived factors related to more keratinocyte apoptosis in depigmented than normally pigmented suctionblistered epidermis may cause passive melanocyte death in vitiligo. J Invest Dermatol 2005;124:976-983.

31 Moretti S, Spallanzani A, Amato L, et al. New insights into the pathogenesis of vitiligo: imbalance of epidermal cytokines at sites of lesions. Pigment Cell Res 2002;15:87-92.

32 Scott G, Cassidy L, Abdel-Malek Z. Alpha-melanocytestimulating hormone and endothelin-1 have opposing effects on melanocyte adhesion, migration, and pp125FAK phosphorylation. Exp Cell Res 1997;237: 19-28.

33 Horikawa T, Norris DA, Yohn JJ, et al. Melanocyte mitogens induce both melanocyte chemokinesis and chemotaxis. J Invest Dermatol 1995;104:256-259. 